\title{
Expert System for Further Training Management in SMEs
}

\author{
Marcel Randermann*, Roland Jochem, Stephan Siek, Thanh Thuy Nguyen \\ Institute for Machine Tools and Factory Management, Technische Universität Berlin, Berlin, Germany. \\ * Corresponding author. Tel.: +49 (0)30 314 23996; email: marcel.randermann@tu-berlin.de \\ Manuscript submitted October 27, 2017; accepted November 9, 2017. \\ doi: 10.17706/ijeeee.2018.8.3.145-153
}

\begin{abstract}
The purpose of this paper is to define and describe an expert system for decision-support in further training management in small and medium-sized enterprises (SME). This will be achieved by evaluating the interrelation of the methodological-didactic characteristics of further training activities on the one hand and, on the other hand, linking them with individual learning preferences in order to recommend high-quality and individual measures when selecting continuing vocational training. The approach takes into account both the teaching-learning-arrangement and the learning style of a learner for algorithm-based decision support. For this purpose inherent characteristics of training activities were determined and interrelations identified by qualitative content analysis of educational literature and expert interviews among training managers of SMEs.
\end{abstract}

Key words: Decision support, further training management, human resource development, methodical-didactic analysis.

\section{Introduction}

Small and medium-sized enterprises (SMEs) face particular challenges in the area of human resource development due to limited resources. Within the scope of a research project of the Department of Quality Science at the Institute for Machine Tools and Factory Management at the Technische Universität Berlin, an approach based on educational science research was developed, which allows the selection of optimal and individual further training activities based on an algorithm. In this paper, the approach is presented and possible opportunities are described and reflected.

In-company continuing education and training as an investment of companies in the knowledge and ability of their employees is now as important as ever. Due to the changes in the technologies, the higher dynamics of the markets and the changes in the value of modern society, vocational further training is becoming more and more important. But also the adjusted attitude towards the employee as a success factor and performance factor rather than as a cost factor has decisively contributed to this [1]. Today, competitive advantages are obtained not only from financial resources or product and process technologies, but above all from technical knowledge and rapid information flows, which are increasingly complex. As a result, the employees' knowledge becomes more and more critical for companies and especially SMEs. Qualified, competent and motivated employees are a key success factor for securing the innovation, performance and competitiveness of companies [2].

From the perspective of the company, further training activities cover the need for company education to maintain and improve entrepreneurial competitiveness and operational potential. Through sustainable management of in-company training, SMEs can preserve, strengthen and develop their core competencies. 
In addition, they can avoid unnecessary personnel changes while generating long-term competitive advantages. Investments in a holistic management of further education pay off twice for SMEs, on the one hand, structured and targeted training facilitates a rapid qualification adjustment of the employees to changed circumstances and situations and on the other hand, the flexibility and mobility of the employees is increased. Furthermore, the satisfaction of the employees and the attractiveness of the employer can be raised. The employees themselves have a greater interest in personal development, because they can make a positive contribution to their own job security through further training. For the employees, continuing vocational training means primarily the securing of their own workplace, but also the possibility to improve their position and their income. Furthermore, they can develop themselves not only professionally but also personally through suitable measures.

Together with the staff recruitment, in-company training forms the central path to further develop knowledge in the company. In particular, SMEs are required to offer their employees the best qualifications due to rapidly changing qualification requirements. However, most SMEs are struggling with limited human, temporal and financial resources in contrast to large companies. In addition, responsible persons in SMEs often lack the necessary know-how in the areas of human resources, educational science as well as information and communication science [3]. They are faced with the challenge to find a further education from the multitude of further training courses which, on the one hand, convey the competencies demanded for the requirements of the company and, on the other hand, meet the requirements and learning preferences of the employees in the company. The research project was designed to develop an effective and efficient approach for the further education management of SMEs that face these challenges.

\section{Postgraduate Professional Education in SMEs}

SMEs form the backbone of the European economy. In 2015, just under 23 million SMEs generated €3.9 (US\$4.6) trillion in value added and employed 90 million people, which equates to two thirds of European employment and slightly less than three fifths of European value added in the non-financial business sector [4].

The concept of human resources development emerged in the 1970s [5] and since then researchers and policy makers have emphasized the importance of investing in the development of human resources in order to improve the quality of human capital and create a sustainable competitive advantage [6]. But when talking about human resource development and company training, there is still a big difference between large companies and SMEs in the 21st century. The size of the company plays a decisive role in the company's participation in workforce development. Data from the OECD countries indicate that SMEs participate $50 \%$ less in training activities than large firms. The fact that SMEs offer considerably fewer employee training than larger firms is in contrast to the growing importance of knowledge and skills intensive occupations in the future [7].

In the EU average between 2005 and 2010 SMEs' rate of training incidence through courses increased by $12.5 \%$ (6.5 percentage points) and it increased by $8 \%$ (3.5 percentage points) in other forms. Participation rates in courses increased by $15.5 \%$ (4.5 percentage points) and the hours in training per employee increased by $17 \%$ (one hour). In summary, it can be said that the training activities of SMEs have developed positively in recent years. Nevertheless, substantial differences in training performance between SMEs and large enterprises persist and require further attention [8].

Against this background, the aim of the project was to develop an application supporting SMEs to increase and improve their human resource development and their further training activities, by bundling the educational science findings and making them approachable to SMEs with the help of an expert system. Our developed methodical approach is intended to help the SME's continuing education management by 
recommending didactical valuable and individualized further training on the basis of an algorithm. The core innovation of the approach is the so-called Education Graph, which takes into account both the teaching-learning-arrangement and the learning style of a learner for algorithm-based decision support.

The decisive aspect of how the Education Graph differs from conventional further education databases is the evaluation of the teaching-learning-arrangement by analyzing the interaction of the methodical-didactic characteristics (e.g. learning form, learning method, social form, educational objectives or learning media) and the adjusting to the learners by identifying the learning type along individual learning preferences and learning styles.

\section{Methodology}

The educational scientific knowledge about training activities and learning styles largely consists of qualitatively justified knowledge. The analytical inductions provide 'soft' real-world data that cannot be generalized. There are many descriptive and interpretive data for understanding interrelations and establishing classifications or typologies but only a few 'hard', recoverable data that are quantitatively justified. In addition, many factors are unstable both in terms of time and context. For this reason and in order to generate all relevant attributes, data from the qualitative content analysis of educational literature were combined with expert surveys. The integration of different methods and the data triangulation provide more valid results.

For the basic development of the algorithm, the didactic-methodological attributes of a further training activity were determined and their interrelations were examined on the one hand and, on the other, the didactic factors were assigned to the learning styles. This results into two distinct measures for identifying fitting training activities.

\subsection{Qualitative Content Analysis and Expert Survey}

For the comparison of further training activities the algorithm requires not only the organization-related (e.g. time and cost frameworks, organizational and legal framework conditions, or circumstances of the learning location) and user-related attributes (e.g. age, professional experience, language) but also the didactic-methodical attributes of further training activities. Based on the structured content analysis of the educational literature the activities were itemized into their individual components and their interrelations were assessed. For this purpose, the attributes were set in an interrelation matrix, respectively graph. In addition, the data from the content analysis were supplemented by the results of the expert interviews.

Learning formats are built of a mixture of methodologically and didactically appropriate methods, media and have different educational objectives. With the data of the interrelation matrices, the algorithm can evaluate the activities with regard to its methodical-didactic conception.

\subsection{Determination of Learning Style}

For the determination of the learning style, a corresponding learning style model in accordance with David A. Kolb [9] was included. The learners are assigned a learning type according to the answers of a questionnaire that is integrated in the expert system. It can be distinguished between four different learning types: Diverging (feeling and watching) - These people are discoverers and learn well through concrete experiences as well as reflected observations. Assimilating (watching and thinking) - People with an assimilating learning style are thinkers and prefer reflected observation, abstract conceptualization, and theoretical models. Converging (doing and thinking) - These people are decision makers and learn well through abstract conceptualization as well as active experimentation. Accommodating (doing and feeling) People with an accommodating style are practitioners who prefer to actively experiment and make concrete experiences. 


\section{Results}

The Education Graph combines the results of the content analysis and the expert survey with the respective learning styles and learning preferences of the learner in order to select optimal and individual further training activities.

\subsection{Formation of Interrelation Matrices}

The defined methodical-didactic components of further training activities include the attributes (Fig. 1): learning forms (LF), teaching and learning methods (TLM), teaching and learning media (LM), learning process (LP), type of mediation (TM), educational objectives (EO) and social form (SF).

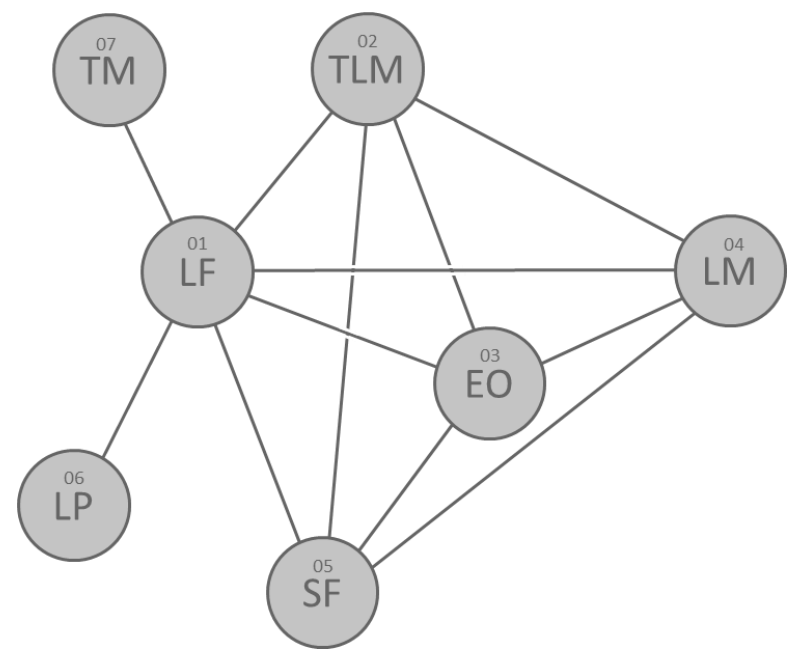

Fig. 1. Interrelation graph of attributes.

In a matrix, the interrelations between the individual attributes more precisely its characteristics were determined on an ordinal scale. For a better understanding, the following is an example of the interrelation between learning form and teaching and learning method. A total of forty different learning forms [10]-[12] and fifty different teaching and learning methods [13]-[16] were determined from the literature. The entire considered data and determined results can be requested from the authors of this paper. The matrix can be used to show how a particular teaching and learning method and a specific learning form interrelate in a training activity. The matrix indicates the appropriateness and degree of verification for the particular interrelation. This differentiation is necessary to maintain a high level of validity with regard to the qualitative and contextual data. The analysis and synthesis of the theoretical and conceptual foundations in the literature as well as the evaluation of the expert survey were used to identify the dominant methodological and didactic approaches, to summarize the most important content and to determine the results of the compilation of further training activities. The indication of appropriateness is determined through extended literature analysis and experts assessment, whereas the degree of verification depends on the number of sources that confirm this certain appraisal. For example, the teaching and learning method 'Pro-Contra-Argumentation' is more appropriate for learning forms such as 'quality circle' and 'blended learning' than for learning forms such as 'model-based learning' or 'instruction' [11], [17], [18].

The results of the content analysis (Table 1) were supplemented by expert surveys to the decision-making processes of personnel developers with regard to the design of training activities (Table 2). Based on Bloom's Taxonomy of Educational Objectives [19] experts have assigned the various learning forms, teaching and learning methods as well as the learning media to the respective six educational objectives (knowledge, comprehension, application, analysis, synthesis, evaluation) in a survey. 
Table 1. Interrelation between Teaching and Learning Methods and Learning Forms

\begin{tabular}{|c|c|c|c|c|c|c|c|c|}
\hline & & & & & & ing forms & & \\
\hline & & & & & 02 & 03 & & 40 \\
\hline & & & & $\begin{array}{c}\text { Blended } \\
\text { Learning }\end{array}$ & Model-based-learning & Coaching & $\cdots$ & Workshop \\
\hline & 01 & Lectur & & $\bullet$ & ० & ० & $\ldots$ & ० \\
\hline$\Xi$ & 02 & Instru & & ० & $\bullet$ & - & $\ldots$ & 0 \\
\hline$\frac{0}{0} \frac{n}{0}$ & 03 & Role $\mathrm{p}$ & & - & 0 & - & $\ldots$ & - \\
\hline$\infty$ & 04 & Partne & ork & $\bullet$ & 0 & & $\ldots$ & ○ \\
\hline ש & & & & $\cdots$ & $\cdots$ & $\cdots$ & $\cdots$ & $\cdots$ \\
\hline & 50 & Meta 1 & technique & - & o & - & $\ldots$ & - \\
\hline Legen & & & & & & & & \\
\hline Appro & riate & ess & & Degre & verification & & & \\
\hline$\bullet \operatorname{mos}$ & appr & priate & - less app & & high number of sources $\mathrm{c}$ & ing the inte & & \\
\hline - very & appr & riate & $\circ$ not appr & & low number of sources $\mathrm{c}$ & ing the inte & & \\
\hline
\end{tabular}

Table 2. Interrelation between Learning Media and Educational Objectives Educational objectives

\begin{tabular}{|c|c|c|c|c|c|c|c|}
\hline & & Knowledge & Comprehension & Application & Analysis & Synthesis & Evaluation \\
\hline \multirow{7}{*}{ 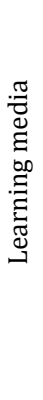 } & Text & $\bullet$ & $\bullet$ & - & $\bullet$ & - & ○ \\
\hline & Image & $\bullet$ & $\bullet$ & $\bullet$ & $\bullet$ & - & 0 \\
\hline & Audio & - & $\bullet$ & $\bullet$ & - & ○ & 0 \\
\hline & Video & $\bullet$ & $\bullet$ & $\circ$ & $\bullet$ & 0 & 0 \\
\hline & Human & $\bullet$ & $\bullet$ & $\bullet$ & $\bullet$ & - & - \\
\hline & Animation/Simulation & $\bullet$ & $\bullet$ & $\bullet$ & - & $\bullet$ & $\bullet$ \\
\hline & Model & $\bullet$ & $\bullet$ & $\bullet$ & - & - & $\bullet$ \\
\hline
\end{tabular}

\section{Legend}

Appropriateness

- most appropriate $\quad$ appropriate not appropriate

- very appropriate $\quad$ less appropriate

\subsection{The Functionality of the Education Graph}

The Education Graph is the core innovation of the research project and bundles the pedagogical-scientific approaches, concepts and findings with regard to high-quality teaching-learning-arrangements of further training activities in an algorithm.

The Education Graph operates in five steps [20]. First, by entering a search criterion, a coarse filtering is made with regard to the required target position. The search criterion can be case-related a competence to be acquired, a topic area or a key concept. In the next step, the users can additionally limit the search result on the basis of organizational framework conditions and formalities. This applies to factors such as time and cost frames, organizational and legal framework conditions, or the desired learning location. This is followed by the evaluation of the teaching-learning arrangement as well as the coordination with the learners along individual learning preferences and learning styles. The options remaining after this filter procedure are displayed so that a manual selection completes the search for the appropriate training offer. For the learner, the Education Graph offers the opportunity to take into account the didactic-methodical 
concept as well as the adjustment to individual learning preferences in the selection process.

\subsection{Evaluation of Teaching-Learning-Arrangement}

To implement the identified and evaluated interrelations in an algorithm-based expert system, the calculation method needs to be defined. Each training activity with its specific characteristics of a set of attributes has a calculated score assigned for relative comparison of various training activities. $f: A \rightarrow S$ with $f\left(c_{1,1}, c_{1,2}, \ldots, c_{n, p}\right)=s$ where $c_{n, 1}, c_{n, 2}, \ldots, c_{n, p} \in a_{n}=\{$ set of characteristics of attribute $n \mid p \in \mathbb{N}\}$ and $S=\{s \mid 0 \leq s \leq 1\}$ as well as $a_{1}, a_{2}, \ldots, a_{n} \in A=\{$ set of attributes $\mid n \in \mathbb{N}\}$. The higher $s$, the more recommended is the training activity compared relatively to similar ones. Each interrelation between characteristics of distinct attributes is assigned a numerical value $I_{c_{n, p} c_{m, q}}$ with $c_{n, p}$ being characteristic $p$ of attribute $n$ and $c_{m, q}$ being characteristic $q$ of attribute $m$ where $c_{n, p} \in a_{n} \in A$ and $c_{m, q} \in a_{m} \in A$ with $n \neq m$. This value considers both the appropriateness and degree of verification through the lower bound of Wilson score confidence interval for a Bernoulli parameter [21].

$$
\begin{gathered}
I_{c_{n, p} c_{m, q}}=\left(\hat{p}+\frac{z_{\alpha / 2}^{2}}{2 n}-z_{\alpha / 2} \sqrt{\left[\hat{p}(1-\hat{p})+z_{\alpha / 2}^{2} / 4 n\right] / n}\right) /\left(1+z_{\alpha / 2}^{2} / n\right) \\
\hat{p}=\text { fraction of sources confirming the } \\
\text { appropriateness of two characteristics } \\
z_{\alpha / 2}=(1-\alpha / 2) \text { quantile of normal distribution } \\
n=\text { total number of sources }
\end{gathered}
$$

There is an $I$ for every $c_{n, p}$ and $c_{m, q}$ if $a_{n}$ and $a_{m}$ inhere an interrelation. Almost every training activity is described by more than two characteristics of disjunctive attributes in a way that several interrelations of characteristics have to be considered. The aggregated score of a training activity is determined by considering the set of inherent characteristics using a recursive function. This approach ensures that each inherent interrelation is weighted according to their occurrence in the graph and significance to the constitution of the training activity. Moreover, activities with varying information depths are normalized for direct comparison.

$$
f_{n}=\left\{\begin{array}{c}
1, \text { if } a_{m}=\varnothing \\
\frac{\sum_{q=1}^{Q} I_{c_{n, p} c_{m, q}}}{\left|a_{m}\right|}, \text { if } a_{m+1}=\varnothing \\
\sum_{m=n+1}^{M}\left(\frac{\sum_{q=1}^{Q}\left(\frac{I_{c_{n, p} c_{m, q}}+I_{c_{n, p} c_{m, q}\left(f_{m}\right)}}{2}\right)}{\left|a_{m}\right|}\right), \text { else }
\end{array}\right.
$$

The recursive function $f$ calculates the teaching-learning-arrangement. The score would be determined by starting the calculation with $f_{n=1}$, where one characteristic of $a_{1}=$ learning form constitutes the training activity. If no interrelation to residual attributes exist, the overall score will be evaluated 1 . Otherwise if merely direct interrelations to knots in the graph exist, the values of the interrelations will be averaged, based on the amount of characteristics of every current attribute. Moreover if the graph of attributes of the specific training activity contains at least three attributes with more than two edges, the recursive function will be called.

Early validation tests, where the algorithm had to rank ten training activity data, showed a $78 \%$ conformance with actual expert's opinions.

\section{Discussion and Implications}


For a holistic application of the Education Graph in operational environment additional components should be utilized. Among these are content management, competence management, and quality management to provide the company a comprehensive and holistic digital support along the course of the continuing education process and to ensure the system-independent use of the approach. A content management should support the user in the systematic recording and administration of further training content and offers as well as training materials in the form of documents, videos or images. The content could be entered manually into the system or transferred from different (internal or external) sources and databases using ETL-processes. A competence management should support the systematic recording and management of the desired and actual key competencies and skills of the person as planning variables for organizational and methodical-didactic program planning. In particular, the focus should be on individual training needs, which must be determined in the course of systematic educational needs analyses. A quality assurance should ensure the quality of the entire training management process as well as knowledge management as the basis for the continuous improvement of in-company training. The interaction of all components would results in a holistic process for the further training management.

If this prerequisite is met, SMEs are able to carry out their continuing vocational training systematically and scientifically founded. Furthermore they can make their further training activities more effective, efficient and resource-conserving. For the employees, the approach offers the possibility of realizing their personal goals, such as the personal desire for knowledge, ability and integration, self-realization and the achievement of professional goals and better career opportunities, both in-house and on the external labor market. By not only finding the best-designed measure for the learner but also a measure that is both well-designed and tailored to the individual's learning level and adapted to individual learning preferences.

\section{Limitations and Further Research Directions}

The remaining need for research is to transfer the developed approach of the expert system and the additionally required components into an operational assistance system. This is then to be tested and optimized, in particular the Education Graph in operational application. Two relevant hurdles are to be named. On the one hand, there is a need for adaptation of the system to the respective competence management system of a company, the construction of which may require a great deal of effort. Furthermore, there are restrictions on data protection compliance regarding the permissible data collection and processing of personal data. On the other hand, the algorithm is based on the current state of scientific research. To a large extent, this knowledge is qualitatively justified and has to be retrieved continually.

From the findings of the validation, corresponding improvement potentials for the assistance system can be identified, analyzed and evaluated as well as success factors for the use of the assistance system. In addition, the research results can serve as the foundation for further innovative projects, such as the further development of the assistance system with regard to machine learning, with which an algorithm can generate the necessary knowledge itself on the basis of statistical evaluations.

\section{References}

[1] Stork, W. (1999). Die Organisation der betrieblichen Weiterbildung. Wiesbaden: Deutscher Universitätsverlag.

[2] Kauffeld, S. (2016). Nachhaltige Personalentwicklung und Weiterbildung. Betriebliche Seminare und Trainings entwickeln, Erfolge messen, Transfer sichern. Heidelberg: Springer-Verlag.

[3] Vollmar, M. (2013). Berufliche Weiterbildung in Unternehmen 2010. Methodik und erste Ergebnisse der CVTS4-Studie. Statistisches Bundesamt, Wirtschaft und Statistik.

[4] European Union. (2016). Annual Report on European SMEs 2015/2016. SME Recovery Continues. 
[5] Hamlin, B., \& Stewart, J. (2011). What is HRD? A definitional review and synthesis of the HRD domain. Journal of European Industrial Training, 35(3), 199-220.

[6] Scheel, T., Rigotti, T., \& Mohr, G. (2014). Training and performance of a diverse workforce. Human Resource Management, 53, 749-772.

[7] OECD. (2013). Skills Development and Training in SMEs, Local Economic and Employment Development (LEED). OECD Publishing.

[8] Cedefop. (2015). Job-Related Adult Learning and Continuing Vocational Training in Europe: A Statistical Picture (Cedefop research paper; No 48.). Luxembourg: Publications Office.

[9] Kolb, D. A. (1985). Learning Style Inventory. Boston: Hay Group, Hay Resources Direct.

[10] Friedrich, A. (2010). Personalarbeit in Organisationen Sozialer Arbeit. Theorie und Praxis der Professionalisierung. Wiesbaden: VS Verlag für Sozialwissenschaften.

[11] Kerres, M. (2013). Mediendidaktik. Konzeption und Entwicklung mediengestützter Lernangebote. München: De Gruyter Oldenbourg.

[12] Schiersmann, C. (2007). Berufliche Weiterbildung. Wiesbaden: VS Verlag für Sozialwissenschaften.

[13] Falk, R. (2000) Betriebliches Bildungsmanagement. Arbeitsbuch für Studium und Praxis. Köln: Wirtschaftsverlag Bachem.

[14] Flechsig, K.-H. (1983). Der Göttinger Katalog Didaktischer Modelle. Theoretische und methodologische Grundlagen. Göttingen: Zentrum für didaktische Studien e. V.

[15] Langosch, I. (1993). Weiterbildung. Planen, Gestalten, Kontrollieren. Stuttgart: Enke.

[16] Rummler, M. (2011). Crashkurs Hochschuldidaktik. Grundlagen und Methoden guter Lehre. Weinheim: Beltz Verlag.

[17] Hallet, W. (2009). Didaktische Kompetenzen. Lehr-und Lernprozesse erfolgreich gestalten. Stuttgart: Klett Lernen und Wissen $\mathrm{GmbH}$.

[18] Stumm, G. \& Pritz, A. (2000). Wörterbuch der Psychotherapie. Wien: Springer Verlag.

[19] Bloom, B. S., Krathwohl, D. R., \& Masia, B. B. (1984). Taxonomy of Educational Objectives: The Classification of Educational Goals. New York: Longman.

[20] Randermann, M., Nguyen T. T. ,\& Jochem, R. (2017). Unterstützung für das Weiterbildungsmanagement in KMU. Ein qualitätswissenschaftlicher Ansatz. Berufsbildung in Wissenschaft und Praxis, 5, 38-39.

[21] Wilson, E. B. (1927). Probable inference, the law of succession, and statistical inference. Journal of the American Statistical Association, 22, 209-212.

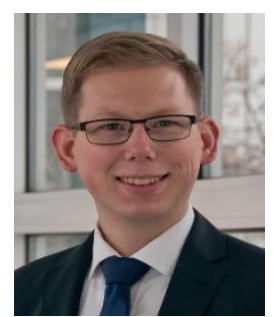

Marcel Randermann was born in September 1989 in Bremen, Germany. He studied industrial engineering at Technische Universität Berlin, Germany and graduated with a bachelor's degree in 2013 and a master's degree in 2017. His major fields of study were production systems and quality management.

During his studies he worked as a self-employed Business Consultant in the field of IT strategy, risk and quality management. Currently he works as a Research Assistant at Department of Quality Science in the Institute for Machine Tools and Factory Management at Technische Universität Berlin and pursues a $\mathrm{PhD}$ degree. His research interests are methodologies of intelligent assistance systems and dynamic reference models in industrial manufacturing.

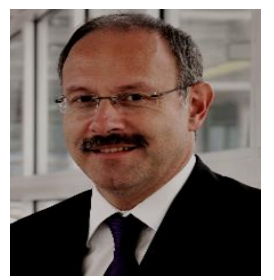

Roland Jochem was born in April 1962. He graduated in mechanical engineering with a diploma degree (Dipl.-Ing.) at Technische Universität Berlin in 1987. His Ph.D. was earned at Technische Universität Berlin in 2002.

Following his job as a research assistant he worked as a group leader process management at Fraunhofer Institute for Production Systems and Design Technology and 
as a process manager for Bosch and Siemens Home Appliances. He was appointed as a professor for quality management at University of Kassel, Germany, University of Stellenbosch, South Africa and Technische Universität Berlin, Germany. Currently he is the director of Institute for Machine Tools and Factory Management and Head of Division Quality Management at Fraunhofer Institute for Production Systems and Design Technology in Berlin, Germany.

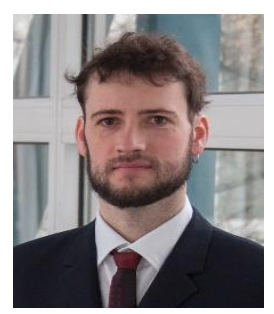

Stephan Siek was born in September 1983 in Stuttgart, Germany. He obtained a diploma degree with computer science of the University of Stuttgart, Germany in 2014. He focused on parallel and intelligent systems in his studies.

During his studies he started working as a software-developer and a system \& network administrator for small and medium-sized enterprises. At the University of Stuttgart he did research on semantic technologies and developed a visual query language to increase the usability for searching related data: QueryVOWL - A Visual Query Notation for Linked Data (ESWC 2015, Portorǒz, Slovenia). Then he joined the department of Quality Science at Technische Universität Berlin working as Research Assistant and researched a configurable plugin-based system architecture to implement software in short time cycle within an interdisciplinary team

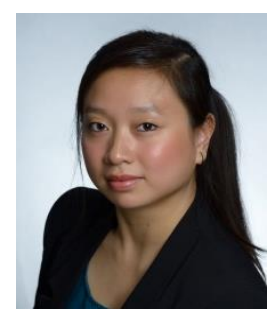

communication.

Thanh Thuy Nguyen was born in July 1989. She received her B.A. degree in culture and technology / philosophy in 2014 and her M.A. degree in educational science organization and consulting from Technische Universität Berlin in 2017.

She is a research assistant at the Institute for Educational Science at Technische Universität Berlin and works on a project of intercultural qualification. Her research interests are education management, science marketing and intercultural 